\title{
Arabic Version of short form of the Speech, Spatial, and Qualities of Hearing Scale (SSQ12)
}

\author{
Mohammad B. Alkhodair, BSc, Tamer A. Mesallam, MD, PhD, Abdulrahman Hagr, MD, FRCSc, Medhat F. Yousef, MD, PhD.
}

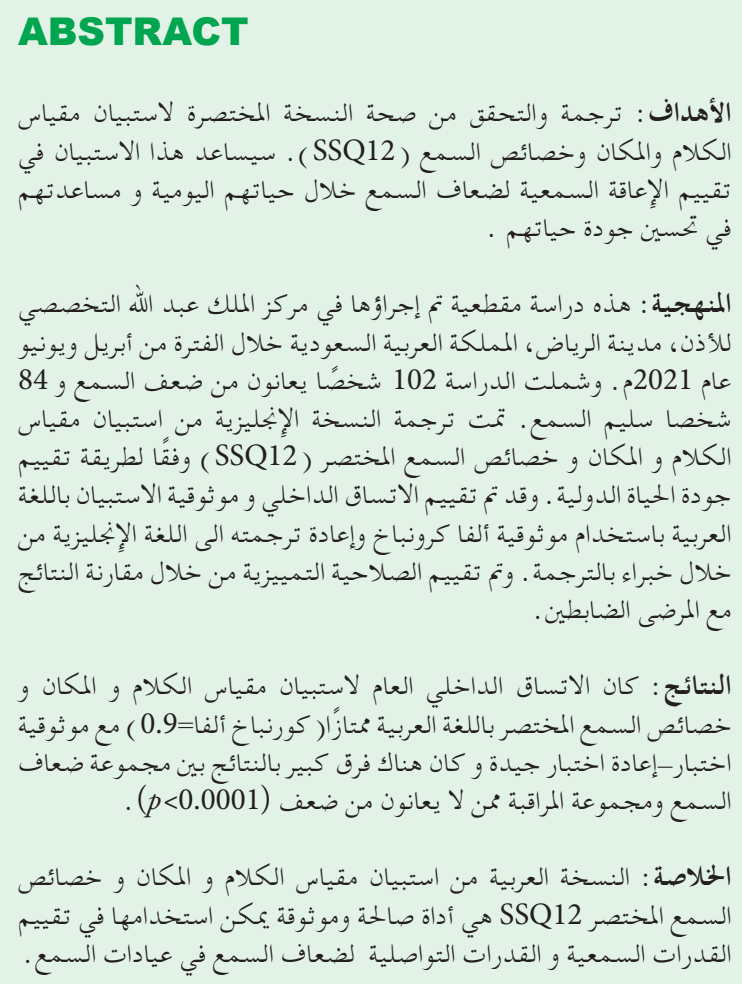

Objectives: To translate and validate the short form of the speech, spatial, and qualities of hearing scale (SSQ12). This will help in the assessment of hearing disability in the daily life of hearing-impaired populations, as well as the improvement of their quality of life.

Methods: This cross-sectional study was conducted at the King Abdullah Ear Specialist Center, Riyadh, Saudi Arabia, between April and June 2021. The study included 102 hearing-impaired participants as the patient group and 84 normal-hearing participants as the control group. The English SSQ12 was translated according to the International Quality of Life Assessment method. Internal consistency and reliability of the Arabic SSQ12 were then assessed using Cronbach's alpha $(\alpha)$ and test-retest reliability.
Discriminant validity was also assessed by comparing the patients' scores with the control participants.

Results: The overall internal consistency of the Arabic SSQ12 was excellent $(\alpha=0.9)$, with good testretest reliability (intraclass correlation coefficient $[\mathrm{ICC}]=0.8)$. There was a significant difference between the Arabic SSQ12 scores of the patient and control groups $(p<0.0001)$.

Conclusion: The Arabic version of the SSQ12 appears to be a valid and reliable tool that can be used to assess the communication ability of hearing-impaired patients in audiology clinics.

Keywords: Hearing impairment, quality of life, the speech, spatial and qualities of hearing scale (SSQ12)

Saudi Med J 2021; Vol. 42 (11): 1180-1185 doi: 10.15537/smj.2021.42.11.20210441

From the Audiology Unit (Alkhodair), King Salman Armed Forces Hospital, Tabuk, from the Research Chair of Voice, Swallowing, and Communication Disorders (Mesallam), Otolaryngology Department, College of Medicine, King Saud University; from King Abdullah Ear Specialist Center (Hagr, Yousef), College of Medicine, King Saud University, Riyadh, Kingdom of Saudi Arabia, and from the Audiology Unit (Yousef), Otolaryngology Department, College of Medicine, Menoufia University, Shebin Alkoum, Egypt.

Received 6th June 2021. Accepted Gth September 2021.

Address correspondence and reprint request to: Dr. Tamer A. Mesallam, Otolaryngology Department, College of Medicine, King Saud University, Riyadh, Kingdom of Saudi Arabia. E-mail: tmesallam@ksu.edu.sa ORCID ID: https://orcid.org/0000-0002-9073-2357

Disclosure. This study was funded by the Deanship of Scientific Research through Vice Deanship of Scientific Research Chairs, King Saud University, Riyadh, Kingdom of Saudi Arabia. 
T he World Health Organization (WHO) estimated that over 430 million people experience disabling hearing loss, which could increase to approximately 700 million by 2050 . If left unaddressed, hearing loss could result to significant challenges in all age groups, including delayed language development, difficult communication and cognition, as well as limited scholastic achievement, employment opportunities, and social engagement. ${ }^{1}$ Furthermore, after assessing the years lived with different disabilities, the Global Burden of Disease Study also reported that hearing loss is the fourth leading cause of disability worldwide. ${ }^{2}$

According to the International Classification of Impairment, Disability and Handicaps, hearing loss should be regarded as a combination of hearing impairment (the dysfunction measurable in the laboratory or clinic), hearing disability (the auditory difficulties experienced by the individual in daily life), and hearing handicap (the non-auditory effects of hearing disability on daily life). ${ }^{3}$ Out of these 3 , only hearing impairment is the measured aspect in routine audiological practice, whereas the other aspects of hearing loss (hearing disability and hearing handicaps) have not been covered. ${ }^{4}$ Fortunately, Gatehouse et al, ${ }^{5}$ published a research on the disabling effects of deficit hearing in 2004, touching on the relevant impacts of those disabilities on the degree of handicap experienced by a hearing-impaired individual. As a result, this research led to the release of the Speech, Spatial, and Qualities of Hearing Scale (SSQ). ${ }^{5}$

To date, the SSQ is now one of the most widely used self-report questionnaires assessing people's abilities in 3 following domains: 1) speech understanding, wherein circumstances including competing sounds, the ability to see other speakers, the number of involved speakers in a conversation, and a variety of background situations (silence, constant noise, contemplation, and a variety of others) were evaluated; 2) spatial domain, in which the ability of a person to process direction, distance, and movement were all assessed; and 3) the hearing quality, where sound separation, recognition, clarity/ naturalness, and listening effort were assessed. ${ }^{5}$

This multi-domain approach in the assessment of hearing-impaired patients has made the SSQ questionnaire a well-qualified tool for the evaluation of communication abilities in these populations. ${ }^{5}$ Moreover, the SSQ has also been used to evaluate the benefits of different rehabilitative interventions, including hearing aids and cochlear implants (CIs). ${ }^{6-9}$ However, although the full 49-item scale of the SSQ is useful in its current form, studies show that an abbreviated version would be useful for regular evaluations and follow-up in the clinical management of hearing disorders. ${ }^{10}$
Numerous studies have used the SSQ 49 for different purposes, such as in the comparison of subjective and objective outcomes following bilateral CIs in adult patients, comparison of age effects on the SSQ results between 2 adult groups with minimal audiometric loss, comparison of CI users with CI plus contralateral linear frequency transposing hearing aids, and assessment of patients with unilateral hearing loss abilities using the SSQ questionnaire. ${ }^{10-13}$ Aside from the original SSQ, short forms of this questionnaire have also been made available. In particular, Demeester et al, ${ }^{4}$ published the SSQ5, which is a useful population screening method that was developed using statistical analyses to achieve a shorter SSQ with the best sensitivity and specificity possible. Similarly, Kiessling et $\mathrm{al}_{,}{ }^{14}$ also established a 15-item short form of the SSQ, which was used in epidemiological settings as a supplement to their binaural hearing function laboratory investigations.

In 2013, Noble et $\mathrm{al},{ }^{15}$ created the SSQ12, which was a clinically useful short form of the original SSQ scale. However, unlike the other short forms, the aim of the SSQ12 was to compile a collection of relevant assessments that represented the scale as a whole, providing clinicians and researchers with a practical scaled-down version. Studies have indeed shown that the SSQ12 provided similar results to SSQ49 in a large clinical research sample. ${ }^{15}$

Recently, the SSQ questionnaire has been translated and validated in many languages worldwide. ${ }^{16-19}$ Among these languages, the Arabic language is one of the 6 official languages of the United Nations. Additionally, Arabic is the official language of 25 countries (Arabic World) with around 422 million speakers, making it the 5th most spoken language in the world.

Considering the wide reach of the Arabic language and its benefit in the evaluation of hearing disability, hearing handicap, and benefits of different rehabilitative interventions, the aim of this study was to translate and validate the SSQ12 in Arabic.

Methods. The study was conducted at the King Abdullah Ear Specialist Center, Riyadh, Saudi Arabia, between April and June 2021 in accordance with the principles of the Declaration of Helsinki and was approved by the Institutional Review Board of the College of Medicine, King Saud University, Riyadh (approval no. E-20-5241). The required sample size was calculated to be 128 (64 in each group), which was based on a required effect size of 0.5 , a $p=0.05$ level of significance, and a power of study of 0.8 . As such, a total of 102 hearing-impaired adult participants (patient group) and 84 normal-hearing adult 
participants (control group) were included in the study. These participants were recruited from the audiology clinic at our facility, whereas control participants were collected from normal-hearing family members with no current or previous history of hearing-related problems. Furthermore, the inclusion criteria for the patient group included the following characteristics: being older than 18 years of age, having a confirmed hearing loss diagnosis, and having Arabic as the native language. Illiterate patients and incomplete answers were therefore excluded from the study.

The original English version of the SSQ12 was translated according to the International Quality of Life Assessment method. ${ }^{20}$ The resulting Arabic translation was back-translated into English and was then compared to the original English version to ensure that there were no major differences. The yielded Arabic SSQ12 was administered to 25 hearing loss patients for pilot testing, and it was further adjusted based on the results. In addition, Dr. William Noble was contacted, and after gaining his approval, the final translated version of the Arabic SSQ12 was administered to both the patient and control groups of the study.

Medical files of all hearing-impaired patients were reviewed. Pure-tone audiometry was performed, and pure-tone averages were calculated using thresholds at 500,100,2000, and $4000 \mathrm{~Hz}$. A soft copy of the Arabic SSQ12 was provided to the participants, and an audiologist was in contact with the participants.

Content validity was used to assess the validity of the Arabic version of SSQ12. The items of the translated questionnaire were judged by an experienced audiologist, with regard to their relevance in the purpose they serve and how culturally appropriate they were to the Arab community. Moreover, concurrent validity was assessed by comparing the scores of the patient and control groups, as well as by the results before and after management in the patient group. For test-retest assessment, the Arabic SSQ12 was re-administered to 30 patients (patient group) 4 weeks after the initial assessment.
Statistical analysis. The Shapiro-Wilk test was used to determine the normal distribution of the data, showing non-normally distributed data. Accordingly, non-parametric statistics were used for data analysis. Internal consistency was measured by testing Cronbach's alpha $(\alpha)$ values of the Arabic SSQ12, and the intraclass correlation coefficient (ICC) was used to assess its test-retest reliability. Discriminant validity was calculated by comparing the SSQ scores of the patient and control groups using the Mann-Whitney U-test, whereas Wilcoxon tests were used to determine the responsiveness rate within the groups by comparing the preoperative and postoperative SSQ results. The Statistical Package for Social Sciences, Version 17 (SPSS Inc., Chicago, IL, USA) software was used for all statistical analyses, and a $p$-value $<0.05$ was considered significant.

Results. This study was conducted in 2 groups: 102 participants in the patient group and 84 participants in the control group. The mean age of the patient group was $32.84 \pm 8.72$ years, comprising 74 males and 28 females, whereas the control group had a mean age of $33.01 \pm 11.43$ years, comprising 61 males and 23 females. Both groups were matched for age and gender distribution (Table 1). During data collection, although we did not notice any comments from the participants denoting inappropriate items with regard to culture, some participants could not answer question 10 due to their lack of musical background, thus leading to answer of "Not applicable." Otherwise, all the remaining questions were clear and answered directly.

Internal consistency of the Arabic SSQ12 was revealed to be excellent, as shown by overall $\alpha=0.94$. In addition, Cronbach's alpha value remained excellent, ranging from 0.93-0.95, even if a question was deleted, resulting to significant item-total correlation coefficient values ranging from 0.34-0.86 (Table 2). Furthermore, test-retest reliability was assessed by studying the correlation between the Arabic SSQ scores of the patients. As a result, the ICC of individual questions and the total score of the Arabic SSQ was found to be 0.8 , indicating good reliability (Table 3 ).

Table 1 - Demographic characteristics of the study groups.

\begin{tabular}{lccc}
\hline Characteristics & Patient group $(\mathbf{n}=\mathbf{1 0 2})$ & Control group $(\mathbf{n}=\mathbf{8 4})$ & $P$-value \\
& \multicolumn{2}{c}{$\mathbf{n}(\%)$} & 0.91 \\
\hline Age, years $($ mean $\pm S D)$ & $32.84 \pm 8.72$ & $33.01 \pm 11.43$ & \\
Gender & $28(27)$ & $23(27)$ & 0.56 \\
Female & $74(73)$ & $61(73)$ & \\
Male & SD: standard deviation \\
\hline
\end{tabular}


The discriminant validity of the Arabic SSQ12 is presented in Table 4. The Mann-Whitney U-test was used to compare the questionnaire ratings of the patient and control groups. On discriminant validity assessment, a significant difference was observed between the patient and control group ratings regarding both the individual questions and total Arabic SSQ12 scores $(p=0.005-0.0001)$, indicating that the Arabic SSQ12 could differentiate between participants in the patient and control groups.

Clinical validity of the Arabic SSQ12 was also assessed by comparing the ratings of the patient group before and after management for their hearing problems.

Table 2 - Internal consistency results of the Arabic speech, spatial, and qualities of hearing scale (SSQ12) questionnaire.

\begin{tabular}{lcc}
\hline SSQ12 & $\begin{array}{c}\text { Cronbach's alpha if item } \\
\text { deleted }\end{array}$ & $\begin{array}{c}\text { Item-total correlation } \\
\text { coefficient }\end{array}$ \\
\hline Q1 & 0.95 & 0.34 \\
Q2 & 0.93 & 0.72 \\
Q3 & 0.94 & 0.77 \\
Q4 & 0.93 & 0.65 \\
Q5 & 0.94 & 0.86 \\
Q6 & 0.94 & 0.80 \\
Q7 & 0.93 & 0.80 \\
Q8 & 0.94 & 0.85 \\
Q9 & 0.94 & 0.82 \\
Q10 & 0.94 & 0.79 \\
Q11 & 0,94 & 0.80 \\
Q12 & 0.93 & 0.86 \\
Total & 0.93 & 1.00 \\
\hline
\end{tabular}

Table 3 - Test-retest reliability of the Arabic speech, spatial, and qualities of hearing scale (SSQ12).

\begin{tabular}{lccc}
\hline SSQ scale & Test-retest correlation & ICC & $P$-value \\
\hline Q1 & & & \\
Q2 & 1.00 & & \\
Q3 & & & \\
Q4 & 0.96 & & \\
Q5 & & & \\
Q6 & & $0.92^{*}$ & $0.0001^{*}$ \\
Q7 & 0.99 & & \\
Q8 & & & \\
Q9 & & & \\
Q10 & & & \\
Q11 & & & \\
Q12 & & & \\
Total & \multicolumn{4}{c}{0.99} & \\
\hline \multicolumn{4}{c}{ ICC: intra-class correlation coefficient } \\
\hline
\end{tabular}

On clinical validity assessment, a significant difference was found between the 2 rating scores $(p=0.01)$. Specifically, their pre-intervention SSQ12 score was $5.33 \pm 2.25$, while their post-intervention rating was $6.41 \pm 1.48$ (Figure 1). Given these findings, significant improvement was observed in the patient group ratings postoperatively, as compared to the preoperative ratings. Additionally, these results indicate the sensitivity of the Arabic-SSQ in detecting any changes in patient responses postoperatively.

Discussion. Self-report questionnaires help audiologists to address the real barriers that hearingimpaired patients face in their daily lives. The main objective of the current study was to provide a reliable tool for evaluating the hearing ability, in addition to behavioral audiological evaluation. The results indicated that the Arabic SSQ12 had excellent internal consistency, showing similar results to those of previous studies in other languages. ${ }^{16,18}$ Cronbach's alpha was reported to be 0.94 , indicating homogeneity and reliability between the questions. The Arabic SSQ12 also showed good repeatability with high test-retest correlations ( $\mathrm{r}=0.92)$, which was in accordance with similar studies showing test-retest reliability coefficients of $0.83-0.88 .^{17,21}$

Hearing ability evaluation in addition to behavioral audiological evaluation is crucial for assessing the degree of disability and handicap. The Arabic version of the SSQ12 particularly showed a significant difference in the rating regarding both individual questions and

Table 4 - Discriminant validity of the speech, spatial, and qualities of hearing scale (SSQ12) questionnaire between the study groups.

\begin{tabular}{lllc}
\hline SSQ scale & Patients & Control & $P$-value \\
& \multicolumn{3}{c}{ Mean \pm SD } \\
Q1 & $6.50 \pm 3.37$ & $6.32 \pm 3.40$ & 0.721 \\
Q2 & $6.61 \pm 3.09$ & $8.98 \pm 1.40$ & 0.0001 \\
Q3 & $4.43 \pm 3.77$ & $7.98 \pm 2.24$ & 0.116 \\
Q4 & $4.57 \pm 3.20$ & $5.33 \pm 3.29$ & \\
Q5 & $4.45 \pm 3.52$ & $7.98 \pm 2.26$ & \\
Q6 & $4.47 \pm 3.35$ & $7.58 \pm 2.33$ & \\
Q7 & $4.48 \pm 3.61$ & $8.19 \pm 1.84$ & \\
Q8 & $4.12 \pm 3.13$ & $6.78 \pm 2.40$ & \\
Q9 & $4.04 \pm 3.13$ & $7.30 \pm 2.40$ & \\
Q10 & $4.69 \pm 3.11$ & $7.15 \pm 2.79$ & \\
Q11 & $3.57 \pm 3.43$ & $6,58 \pm 3.10$ & \\
Q12 & $4.24 \pm 3.37$ & $7.16 \pm 2.62$ & \\
Total & $4.69 \pm 2.53$ & $7.28 \pm 1.37$ & \\
\hline \multicolumn{5}{c}{ SD: standard deviation } \\
\hline
\end{tabular}




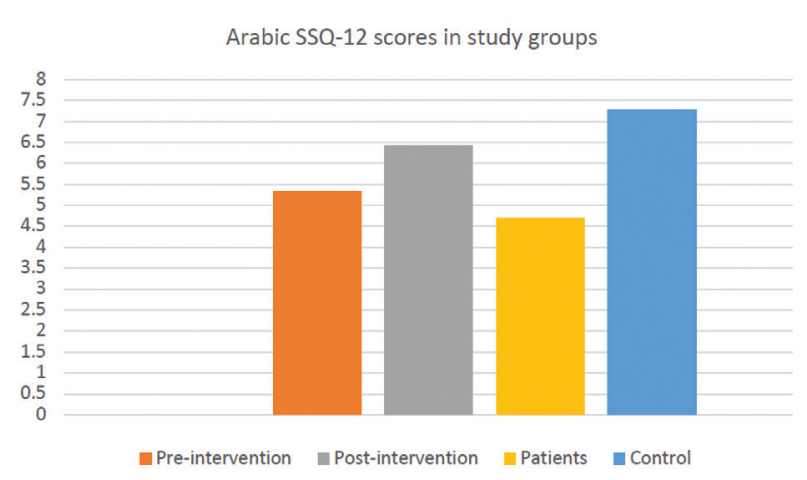

Figure 1 - Comparison between the Arabic speech, spatial, and qualities of hearing scale (SSQ12) scores among the study groups.

total scores between the 2 groups, reflecting that the questionnaire can differentiate between the normal and hearing-impaired individuals. Moreover, our data corresponded with the different SSQ ratings of hearing-impaired and normal-hearing individuals studied by Gatehouse et $\mathrm{al}^{5}{ }^{5}$ and Demeester et al. ${ }^{4,12}$ Arabic SSQ12 administration after any intervention was also found to be very beneficial, as it assessed the benefits that the hearing-impaired patients received from their management. Furthermore, the translated questionnaire showed a high sensitivity in detecting any changes in patient performance when using hearing amplification devices by comparing the results of the hearing-impaired participants before and after using these devices.

The translation and validation of the SSQ12 to the Arabic language will allow hearing-impaired Arabic speakers to express their challenges in particular situations. Moreover, the Arabic SSQ12 could help Arabic-speaking audiologists to further understand the full picture of how hearing loss impacts their clients and use it as a complement to the behavioral and experimental measures of the hearing ability.

Study limitations. Despite these findings, the relatively small sample size and the fact that the study cohort included only sensorineural hearing loss patients are limitations that were noted. Therefore, further studies using the Arabic SSQ12, including other types of hearing loss, can confirm the clinical validity of this tool.

In conclusion, the Arabic SSQ12 is a reliable and valid tool that allows hearing-impaired Arabic speakers to express the different challenges of hearing disability. In addition, the Arabic SSQ12 could help Arabicspeaking audiologists to understand the full picture of how hearing loss impacts their clients and to use it as a valuable tool with other audiological assessments in the evaluation and monitoring of the hearing status among different populations.

Acknowledgment. The authors are grateful to the Deanship of Scientific Research, king Saud University for funding through Vice Deanship of Scientific Research Chairs. We would like to thank Editage (www.editage.com) for English language editing.

\section{References}

1. World Health Organization. World report on hearing [Internet]. 2017 [accessed 2021 March 3]. Available from: https://www. who.int/publications/i/item/world-report-on-hearing

2. Vos T, Allen C, Arora M, Barber RM, Bhutta ZA, Brown A, et al. Global, regional, and national incidence, prevalence, and years lived with disability for 310 diseases and injuries, 1990-2015: a systematic analysis for the global burden of disease study 2015. Lancet 2016; 388: 1545-1602.

3. Thuriaux MC. The ICIDH: evolution, status, and prospects. Disabil Rehabil 1995; 17: 112-118.

4. Demeester K, Topsakal V, Hendrickx JJ, Fransen E, van Laer L, Van Camp G, et al. Hearing disability measured by the speech, spatial, and qualities of hearing scale in clinically normal-hearing and hearing-impaired middle-aged persons, and disability screening by means of a reduced SSQ (the SSQ5). Ear Hear 2012; 33: 615-616.

5. Gatehouse S, Noble W. The speech, spatial and qualities of hearing scale (SSQ). Int J Audiol 2004; 43: 85-99.

6. Martin TP, Lowther R, Cooper H, Holder RL, Irving RM, Reid AP, et al. The bone-anchored hearing aid in the rehabilitation of single-sided deafness: experience with 58 patients. Clin Otolaryngol 2010; 35: 284-290.

7. Noble W, Gatehouse S. Effects of bilateral versus unilateral hearing aid fitting on abilities measured by the speech, spatial, and qualities of hearing scale (SSQ). Int J Audiol 2006; 45: 172-181.

8. Noble W, Tyler R, Dunn C, Bhullar N. Unilateral and bilateral cochlear implants and the implant-plus-hearing-aid profile: comparing self-assessed and measured abilities. Int $J$ Audiol 2008; 47: 505-514.

9. van Wieringen A, De Voecht K, Bosman AJ, Wouters J. Functional benefit of the bone-anchored hearing aid with different auditory profiles: objective and subjective measures. Clin Otolaryngol 2011; 36: 114-120.

10. Hua H, Johansson B, Jönsson R, Magnusson L. Cochlear implant combined with a linear frequency transposing hearing aid. J Am Acad Audiol 2012; 23: 722-732.

11. Laske RD, Veraguth D, Dillier N, Binkert A, Holzmann D, Huber AM. Subjective and objective results after bilateral cochlear implantation in adults. Otol Neurotol 2009; 30: 313-318.

12. Banh J, Singh G, Pichora-Fuller MK. Age affects responses on the speech, spatial, and qualities of hearing scale (SSQ) by adults with minimal audiometric loss. J Am Acad Audiol 2012; 23: 81-91.

13. Teen O, Lotte H, Lars N. Self-reported hearing performance among subjects with unilateral sensorineural hearing loss. Audiol Med 2012; 10: 83-92. 
14. Kiessling J, Grugel L, Meister H, Meis M. [Übertragung der Fragebögen SADL, ECHO und SSQ ins Deutsche und deren Evaluation]. Zeitschrift für Audiologie (Audiological Acoustics) 2011; 50: 6-16. [German]

15. Noble W, Jensen NS, Naylor G, Bhullar N, Akeroyd MA. A short form of the speech, spatial and qualities of hearing scale suitable for clinical use: the SSQ12. Int J Audiol 2013; 52: 409-412.

16. Kong TH, Park YA, Bong JP, Park SY. Validation of the Korean version of the spatial hearing questionnaire for assessing the severity and symmetry of hearing impairment. Yonsei Med J 2017; 58: 842-847.

17. Lotfi Y, Nazeri AR, Asgari A, Moosavi A, Bakhshi E. Iranian version of speech, spatial, and qualities of hearing scale: a psychometric study. Acta Med Iran 2016; 54: 756-764.
18. Moulin A, Richard C. Validation of a French-language version of the spatial hearing questionnaire, cluster analysis and comparison with the speech, spatial, and qualities of hearing scale. Ear Hear 2016; 37: 412-423.

19. Tufatulin GS, Artyushkin SA. [Validation of the Russian language version of the SSQ questionnaire]. Vestn Otorinolaringol 2016; 81: 17-22. [Russian]

20. Bullinger M, Alonso J, Apolone G, Leplège A, Sullivan M, Wood-Dauphinee $S$, et al. Translating health status questionnaires and evaluating their quality: the IQOLA project approach. International quality of life assessment. J Clin Epidemiol 1998; 51: 913-923.

21. Singh G, Kathleen Pichora-Fuller M. Older adults' performance on the speech, spatial, and qualities of hearing scale (SSQ): test-retest reliability and a comparison of interview and selfadministration methods. Int J Audiol 2010; 49: 733-740. 